Thomas S. Salisbury* and Juris Steprāns † Department of Mathematics, York University, 4700 Keele Street, North York, Ontario, Canada M3J 1P3.

e-mail: SaH@@nexus.yorku.ca and Juris.Steprans@@mathstat. yorku.ca

\title{
HAUSDORFF CAPACITY AND LEBESGUE MEASURE
}

\begin{abstract}
It is shown that for any $r \in(0,1)$ and for any continuous function from the unit interval to itself, there are sets of arbitrarily small Lebesgue measure whose preimage has arbitrarily large $r$-Hausdorff capacity. This is generalized to functions from the unit square to the interval.
\end{abstract}

\section{Introduction}

This paper is devoted to establishing what may be viewed as a strong failure of topological invariance between two capacities, one being Lebesgue measure and and the other Hausdorff capacity. In particular, it will be shown that no continuous function can preserve capacity between these two capacities and, indeed, the worst possible failure always occurs. If $r \in[0,1]$, then for any set $X \subseteq[0,1]$ the $r$-Hausdorff capacity of $X$ is denoted by $H_{\infty}^{r}(X)$ and is defined to be the infimum of all $t$ such that there is a cover of $X$ by intervals, $X \subseteq \bigcup_{i=0}^{\infty} I_{i}$, such that $t=\sum_{i=0}^{\infty} \operatorname{diam}\left(I_{i}\right)^{r}$. In other words,

$$
H_{\infty}^{r}(X)=\inf \left\{\sum_{i}\left(\operatorname{diam}\left(I_{i}\right)\right)^{r}: X \subseteq \bigcup_{i} I_{i}\right\}
$$

where the infimum is taken over families of intervals $\left\{I_{i}\right\}_{i=0}^{\infty}$. This notion may be familiar from its use along the way to defining $r$-Hausorff measure.

Key Words: Hausdorff capacity, Lebesgue measure, continuous function

Mathematical Reviews subject classification: Primary 28A12 28A35 28A78; Secondary 26A03 26A15

Received by the editors February 1, 1996

* Partially supported by NSERC of Canada

${ }^{\dagger}$ Partially supported by NSERC of Canada 
Given $\beta>0, H_{\beta}^{r}(X)$ is defined, for any set $X \subseteq[0,1]$, to be the infimum of all $t$ such that there is a cover of $X$ by intervals, $X \subseteq \bigcup_{i=0}^{\infty} I_{i}$, such that $t=\sum_{i=0}^{\infty} \operatorname{diam}\left(I_{i}\right)^{r}$ and such that the length of each interval $I_{i}$ is less than $\beta$. The $r$-Hausdorff measure of a set $X$ is then defined to be the supremum of $H_{\beta}^{r}(X)$ as $\beta$ ranges over all positive real numbers. However, $r$-Hausdorff capacity rather than $r$-Hausdorff measure is the topic of this paper. The crucial difference between the two is that, while the $r$-Hausdorff measure is countably additive, the $r$-Hausdorff capacity is only subadditive if $r \in(0,1)$. For a discussion of capacities in general and the Hausdorff capacity in particular see, for example, Section 30 of [2]. A proof of the fact that $H_{\infty}^{r}$ is actually a capacity can be found in [1].

For the rest of this paper let $r$ be a fixed real number such that $0<r<1$. Let $\lambda(X)$ denote the Lebesgue measure of any measurable set $X \subseteq[0,1]$. It will be shown that, for any $\epsilon>0$ and any continuous function $F:[0,1] \rightarrow[0,1]$ there is $A \subseteq[0,1]$ such that $\lambda(A)<\epsilon$ and such that $H_{\infty}^{r}\left(F^{-1} A\right)>1-\epsilon$. Indeed, the same is true for the Fubini product of $r$-Hausdorff capacity, in spite of the fact that no Fubini type of theorem is available for these capacities. In order to state this result more precisely some notation will be introduced. If $A \subseteq[0,1]^{2}$ and $x \in[0,1]$, then $A_{x}$ will denote $\{y \in[0,1]:(x, y) \in A\}$. On the other hand, if $F: D \rightarrow[0,1]$ is a function with domain $D \subseteq[0,1]^{2}$ and $x \in[0,1]$, then $F_{x}$ will denote the function whose domain is $D_{x}$ and which is defined by $F_{x}(y)=F(x, y)$.

The main theorem can now be stated. It is shown in Theorem 3.1 that if $F:[0,1]^{2} \rightarrow[0,1]$ is any continuous function and $\epsilon>0$, then there is $A \subseteq[0,1]$ such that $\lambda(A)<\epsilon$ and such that

$$
H_{\infty}^{r}\left\{x \in[0,1]: H_{\infty}^{r}\left(F_{x}^{-1} A\right)>1-\epsilon\right\}>1-\epsilon .
$$

While this is interesting on its own because it illustrates a striking difference between the capacity $H_{\infty}^{r}$ and Lebesgue measure, this result is also extended in [4] to serve a different purpose. It is shown there that it is possible to extend a model of set theory so that the ground model set of reals has Lebesgue measure 0 but positive $r$-Hausdorff measure. This implies that the set also has positive $r$-Hausdorff capacity because it is known that $r$-Hausdorff measure and $r$-Hausdorff capacity have the same null sets.

\section{An Observation about Hausdorff Capacity}

The key fact about Hausdorff capacity that will be used is that if $B \subseteq[0,1]$ is of small Lebesgue measure but evenly distributed through [0,1], then $H_{\infty}^{r}(B)$ is close to 1 . This is made precise in the next lemma whose statement requires the following notation. 
Definition 2.1 For any measurable set $A \subseteq[0,1]$ and $i \leq m$ define $\Delta_{m}^{i}(A)$ to be the least real number such that $\lambda\left(A \cap\left[0, \Delta_{m}^{i}(A)\right]\right)=\frac{i \lambda(A)}{m}$.

Notice that $\Delta_{m}^{i}(A)$ is always defined and that if $A=[0,1]$, then $\Delta_{m}^{i}(A)$ is nothing more than $\frac{i}{m}$.

Lemma 2.1 Let $r \in(0,1)$. For each $\mu>0$ and $m \in \mathbb{N}$ satisfying

- $\frac{m^{1-r} \mu}{4 \cdot 2^{r}}>1$

- $\frac{m \mu}{4}\left(\frac{\mu}{2 m}\right)^{r}>1$

if $D \subseteq U \subseteq[0,1]$ are measurable sets such that for each $i<m$

$$
\lambda\left(D \cap\left[\Delta_{m}^{i}(U), \Delta_{m}^{i+1}(U)\right]\right) \geq \frac{\mu}{2 m},
$$

then $H_{\infty}^{r}(D) \geq \lambda(U)-\mu$.

Proof. Let $D \subseteq \bigcup_{i=0}^{\infty} I_{i}$ and suppose that $\sum_{i=0}^{\infty}\left(\lambda\left(I_{i}\right)\right)^{r}<\lambda(U)-\mu$. Let $B=$ $\left\{i \in \mathbb{N}: \lambda\left(I_{i}\right) \geq \frac{1}{2 m}\right\}$ and let $C=\left\{i<m:(\forall j \in B)\left(I_{j} \cap\left[\Delta_{m}^{i}(U), \Delta_{m}^{i+1}(U)\right] \cap\right.\right.$ $U=\emptyset\}$. Three separate cases, depending on the size of $B$ and $C$, will be considered.

Case 1 To begin, suppose that $|B| \geq \frac{m \mu}{4}$. Then

$$
\sum_{i=1}^{\infty} \lambda\left(I_{i}\right)^{r} \geq \sum_{i \in B} \lambda\left(I_{i}\right)^{r} \geq|B|(1 / 2 m)^{r} \geq \frac{m^{1-r} \mu}{4 \cdot 2^{r}}>1
$$

Since $\sum_{i=0}^{\infty}\left(\lambda\left(I_{i}\right)\right)^{r}<\lambda(U)-\mu<1$ it follows that this is impossible.

Case 2 Suppose now that $|B|<\frac{m \mu}{4}$ and $|C| \leq \frac{m \mu}{2}$. It then follows that if

$$
G=\left\{i<m:\left[\Delta_{m}^{i}(U), \Delta_{m}^{i+1}(U)\right] \cap U \nsubseteq \bigcup_{j=1}^{\infty} I_{j}\right\},
$$

then $|G| \leq 2 \cdot|B|+|C|$. The reason for this is that if $i \in B$, then there are at most two integers $j$ such that the intervals $\left[\Delta_{m}^{i}(U), \Delta_{m}^{i+1}(U)\right]$ intersect $I_{i}$ but are not contained in $I_{i}$ - this accounts for the summand $2 \cdot|B|$. All the other intervals which could be included in $G$ must be disjoint from $I_{i}$ for every $i \in B$ - this accounts for the other summand $|C|$.

By the assumptions of this case it follows that $2 \cdot|B|+|C|<m \mu$ and hence

$$
\lambda\left(U \backslash\left(\bigcup_{i \in G}\left[\Delta_{m}^{i}(U), \Delta_{m}^{i+1}(U)\right] \cap U\right)\right)
$$


is at least as great as $\lambda(U)-\mu$. Since

$$
U \backslash\left(\bigcup_{i \in G}\left[\Delta_{m}^{i}(U), \Delta_{m}^{i+1}(U)\right] \cap U\right) \subseteq \bigcup_{i=1}^{\infty} I_{i}
$$

this yields that $\sum_{i=1}^{\infty}\left(\lambda\left(I_{i}\right)\right)^{r} \geq \sum_{i=1}^{\infty} \lambda\left(I_{i}\right) \geq \lambda(U)-\mu$ which is again a contradiction.

Case 3 Suppose that $|B|<\frac{m \mu}{4}$ and $|C|>\frac{m \mu}{2}$. Let $C^{\prime}$ be a family of nonconsecutive members of $C$ of maximal cardinality - hence, $\left|C^{\prime}\right| \geq|C| / 2>\frac{m \mu}{4}$. Let

$$
E_{j}=\left\{i \in \mathbb{N}: I_{i} \cap\left[\Delta_{m}^{j}(U), \Delta_{m}^{j+1}(U)\right] \cap U \neq \emptyset\right\}
$$

for each $j \in C^{\prime}$ and let $E=\bigcup_{j \in C^{\prime}} E_{j}$. Since, for $j \in C$, the sets

$$
\left[\Delta_{m}^{j}(U), \Delta_{m}^{j+1}(U)\right] \cap U
$$

are intersected only by intervals $I_{i}$ where $i \in \mathbb{N} \backslash B$, and such intervals $I_{i}$ are smaller than any $\Delta_{m}^{j+1}(U)-\Delta_{m}^{j}(U)$, it follows that $E_{j} \cap E_{k}=\emptyset$ if $k$ and $j$ are distinct members of $C^{\prime}$. Therefore, using the fact that $0<r<1$,

$$
\begin{gathered}
\sum_{i \in E}\left(\lambda\left(I_{i}\right)\right)^{r} \geq \sum_{j \in C^{\prime}} \sum_{i \in E_{j}}\left(\lambda\left(I_{i}\right)\right)^{r} \geq \\
\sum_{j \in C^{\prime}}\left(\sum_{i \in E_{j}} \lambda\left(I_{i}\right)\right)^{r} \geq \sum_{j \in C^{\prime}}\left(\lambda\left(D \cap\left[\Delta_{m}^{j}(U), \Delta_{m}^{j+1}(U)\right]\right)\right)^{r} \geq \\
\sum_{i \in C^{\prime}}\left(\frac{\mu}{2 m}\right)^{r} \geq \frac{m \mu}{4}\left(\frac{\mu}{2 m}\right)^{r}>1
\end{gathered}
$$

and once again, as in the first case, this is a contradiction because $D \subseteq[0,1]$.

\section{Hausdorff Capacity and Continuous Functions}

If $X \subseteq[0,1]$, then $F: X \rightarrow[0,1]$ will be said to have small fibers if and only if $\lambda\left(F^{-1}\{x\}\right)=0$ for each $x \in[0,1]$. The proof of Theorem 3.1 and the lemmas preceding it will rely on decomposing an arbitrary continuous function into two pieces, one of which has small fibers and the other of which has countable range. 
Lemma 3.1 Let $\mu>0$ and suppose that $\left\{X_{i}: i \in \mathbb{N}\right\}$ is a sequence of mutually independent $\{0,1\}$-valued random variables with mean $\mu$ for each $i \in \mathbb{N}$. Suppose also that $C \subseteq[0,1]$ is Lebesgue measurable and that $F: C \rightarrow[0,1]$ is measurable with small fibers. For any $\epsilon>0$ there is $M \in \mathbb{N}$ such that for all $m \geq M$ the probability that

$$
\lambda\left(\cup_{i<m} F^{-1}\left[\frac{i}{m}, \frac{i+X_{i}}{m}\right]\right)>\frac{\mu \lambda(C)}{2}
$$

is greater than $1-\epsilon$.

Proof. Let $m \in \mathbb{N}$ be fixed. Let $\theta_{i}=\lambda\left(F^{-1}\left[\frac{i}{m}, \frac{i+1}{m}\right]\right)$ and let

$$
Y_{i}=\lambda\left(F^{-1}\left[\frac{i}{m}, \frac{i+X_{i}}{m}\right]\right)
$$

and note that the $Y_{i}$ are still independent random variables with value either 0 or $\theta_{i}$. Hence, $\alpha_{m}$, the average value of $\sum_{i<m} Y_{i}$, is the sum of the averages of each $Y_{i}$; namely $\sum_{i<m} \theta_{i} \mu=\mu \lambda(C)$. Similarly, $\sigma_{m}^{2}$, the square of the variance of $\sum_{i<m} Y_{i}$ is the sum of the squared variances of each $Y_{i}$. Hence

$$
\sigma_{m}^{2}=\sum_{i<m}\left(\left(\theta_{i}-\theta_{i} \mu\right)^{2} \mu+\left(0-\theta_{i} \mu\right)^{2}(1-\mu)\right)
$$

Now let $\eta_{m}=\max _{i<m} \theta_{i}$ and note that

$$
\sigma_{m}^{2}=\sum_{i<m} \theta_{i}^{2}(1-\mu) \mu \leq \eta_{m} \sum_{i<m} \theta_{i}(1-\mu) \mu \leq \eta_{m} \sum_{i<m} \theta_{i}
$$

and this final expression is equal to $\eta_{m} \lambda(C)$. It now follows from Chebyshev's Inequality that the probability that $\left|\sum_{i<m} Y_{i}-\alpha_{m}\right|>\frac{\mu \lambda(C)}{2}$ is less than

$$
\sigma_{m}^{2}\left(\frac{2}{\mu \lambda(C)}\right)^{2} \leq \eta_{m} \lambda(C)\left(\frac{2}{\mu \lambda(C)}\right)^{2}
$$

and hence, if $M$ can be chosen so that $m \geq M$ implies that $\eta_{m}<\frac{\epsilon \mu^{2} \lambda(C)}{4}$, then it will follow that the probability that

$$
\lambda\left(\cup_{i<m} F^{-1}\left[\frac{i}{m}, \frac{i+X_{i}}{m}\right]\right)>\frac{\mu \lambda(C)}{2}
$$

is greater than $1-\epsilon$.

To see that $M$ with the required property can be chosen, notice that for each $x \in[0,1]$ there is $\delta_{x}>0$ such that $\lambda\left(F^{-1}\left[x-\delta_{x}, x+\delta_{x}\right]\right)<\frac{\epsilon \mu^{2} \lambda(C)}{4}$ because $F$ has small fibers. Now apply compactness to find a finite subcover

$$
\left\{\left[x_{1}-\delta_{x_{1}}, x_{1}+\delta_{x_{1}}\right],\left[x_{2}-\delta_{x_{2}}, x_{2}+\delta_{x_{2}}\right] \ldots\left[x_{j}-\delta_{x_{j}}, x_{j}+\delta_{x_{j}}\right]\right\}
$$


and let $M$ be so large that for each $i<M$ there is some $n \leq j$ such that $\left[\frac{i}{M}, \frac{i+1}{M}\right] \subseteq\left[x_{n}-\delta_{x_{n}}, x_{n}+\delta_{x_{n}}\right]$.

Lemma 3.2 Let $\mu>0$ and suppose that $\left\{X_{i}: i \in \mathbb{N}\right\}$ is a sequence of mutually independent $\{0,1\}$-valued random variables with mean $\mu$ for each $i \in \mathbb{N}$. Suppose that $C \subseteq[0,1]$ is a measurable set and that for each $j<n$ the function $F_{j}: C \rightarrow[0,1]$ is measurable with small fibers. For any $\epsilon>0$ there is $M \in \mathbb{N}$ such that for all $m \geq M$ the probability that

$$
\lambda\left(\bigcap_{j \in n} \bigcup_{i<m} F_{j}^{-1}\left[\frac{i}{m}, \frac{i+X_{i}}{m}\right]\right)>\left(\frac{\mu}{2 n}\right)^{n} \lambda(C)
$$

is greater than $1-\epsilon$.

Proof. Proceed by induction on $n$ noting that the case $n=1$ is just Lemma 3.1. Given functions $F_{j}: C \rightarrow[0,1]$ for $j<n+1$, first let $\left\{Y_{i}: i \in \mathbb{N}\right\}$ and $\left\{W_{i}: i \in \mathbb{N}\right\}$ be sequences of mutually independent $\{0,1\}$-valued random variables such that

- the probability that $Y_{i}=1$ is $\frac{n \mu}{n+1}$ for each $i \in \mathbb{N}$

- the probability that $W_{i}=1$ is $\frac{\mu}{n+1}$ for each $i \in \mathbb{N}$

- $Y_{i}+W_{i}=X_{i}$ for each $i \in \mathbb{N}$.

Next, given $\epsilon>0$ use the induction hypothesis to find $M^{\prime}$ such that if $m \geq M^{\prime}$, then the probability that

$$
\lambda\left(\bigcap_{j<n} \bigcup_{i<m} F_{j}^{-1}\left[\frac{i}{m}, \frac{i+Y_{i}}{m}\right]\right)>\left(\frac{n \mu}{2(n+1) n}\right)^{n} \lambda(C)
$$

is greater than $1-\epsilon / 2$. Let

$$
Z\left(Y_{0}, Y_{1}, \ldots, Y_{m-1}\right)=\bigcap_{j<n}\left(\cup_{i<m} F_{j}^{-1}\left[\frac{i}{m}, \frac{i+Y_{i}}{m}\right]\right)
$$

and use Lemma 3.1 to find $M^{\prime \prime} \in \mathbb{N}$ such that if $m \geq M^{\prime \prime}$, then the probability that the Lebesgue measure of

$$
\left.\bigcup_{i<m}\left(F_{n} \uparrow Z\left(Y_{0}, Y_{1}, \ldots, Y_{m-1}\right)\right)^{-1}\left[\frac{i}{m}, \frac{i+W_{i}}{m}\right]\right)
$$

is greater than $\frac{\mu}{2(n+1)} \lambda\left(Z\left(Y_{0}, Y_{1}, \ldots, Y_{m-1}\right)\right)$ is greater than $1-\epsilon / 2$. 
Since $Y_{i}+W_{i}=X_{i}$ for each $i \in \mathbb{N}$ it follows that the probability that

$$
\lambda\left(\bigcap_{j<n+1}\left(\bigcup_{i<m} F_{j}^{-1}\left[\frac{i}{m}, \frac{i+X_{i}}{m}\right]\right)\right)>\left(\frac{\mu}{2(n+1)}\right)^{n+1} \lambda(C)
$$

is the same as the probability that

$$
\left.\lambda\left(\bigcap_{j<n+1} \bigcup_{i<m} F_{j}^{-1}\left[\frac{i}{m}, \frac{i+Y_{i}+W_{i}}{m}\right)\right]\right)>\left(\frac{\mu}{2(n+1)}\right)^{n+1} \lambda(C)
$$

and this, in turn, is no smaller than the probability that both of the following happen simultaneously:

- $\lambda\left(Z\left(Y_{0}, Y_{1}, \ldots, Y_{m-1}\right)\right)>\left(\frac{\mu}{2(n+1)}\right)^{n+1} \lambda(C)$

- the Lebesgue measure of

$$
\left.\bigcup_{i<m}\left(F_{n} \uparrow Z\left(Y_{0}, Y_{1}, \ldots, Y_{m-1}\right)\right)^{-1}\left[\frac{i}{m}, \frac{i+W_{i}}{m}\right]\right)
$$

is greater than $\frac{\mu}{2(n+1)} \lambda\left(Z\left(Y_{0}, Y_{1}, \ldots, Y_{m-1}\right)\right)$.

This is no smaller than $(1-\epsilon / 2)(1-\epsilon / 2)>1-\epsilon$.

Lemma 3.3 Given $\epsilon>0$ and $k \in \mathbb{N}$ there is $\delta(\epsilon, k)>0$ such that for any family of measurable functions with small fibers $\left\{F_{i}: C_{i} \rightarrow[0,1]\right\}_{i<k}$, any measurable set $U \subseteq[0,1]$ such that $C_{i} \subseteq U$ and any $\rho>0$ there is $M \in \mathbb{N}$ such that for all $m>M$ and for all $i<k$, for any mutually independent, $\{0,1\}$-valued random variables $\left\{X_{i}\right\}_{i<k}$ with mean $\epsilon / 2$, the probability that

$$
H_{\infty}^{r}\left(\bigcap_{i \in k}\left(F_{i}^{-1} \bigcup_{j \in m}\left[\frac{j}{m}, \frac{j+X_{j}}{m}\right]\right) \cup\left(U \backslash C_{i}\right) \backslash Y\right)>\lambda(U)-\epsilon / 2
$$

for any $Y \subseteq[0,1]$ such that $\lambda(Y)<\delta(\epsilon, k) \lambda(U)$ is greater than $1-\rho$.

Proof. Let $\mu=\left(\frac{\epsilon}{4 k}\right)^{k}$ and use Lemma 2.1 to find $p$ such that if $D \subseteq U$ is a measurable set such that for each $i<p$

$$
\left(\lambda\left(D \cap\left[\Delta_{p}^{i}(U), \Delta_{p}^{i+1}(U)\right]\right)\right)^{r} \geq \frac{\mu}{2 p},
$$

then $H_{\infty}^{r}(D) \geq \lambda(U)-\mu$. Let $0<\delta=\delta(\epsilon, k)<\frac{\mu}{p 2^{k+1}}$. Let $\left\{P_{i}: i<s\right\}$ enumerate the coarsest partition of $U$ refining each of the partitions

$$
\left\{\left[\Delta_{p}^{i}(U), \Delta_{p}^{i+1}(U)\right] \cap U: i<p\right\} \text { and }\left\{C_{i} \cap U, U \backslash C_{i}\right\}
$$


for $i<k$. Now use Lemma 3.2 to find $M \in \mathbb{N}$ such that for all $m \geq M$ the probability that

$$
\lambda\left(\bigcap_{j<k}\left(F_{j}\left\lceil P_{n}\right)^{-1} \bigcup_{i<m}\left[\frac{i}{m}, \frac{i+X_{i}}{m}\right]\right)>\left(\frac{\epsilon}{4 k}\right)^{k} \lambda\left(P_{n}\right)\right.
$$

is greater than $1-\frac{\rho}{s+2}$ for each $n<s$. By making $m$ larger, if necessary, it also possible to ensure that the probability that

$$
\left|\left\{i<m: X_{i}=1\right\}\right|<\epsilon m
$$

is also greater than $1-\frac{\rho}{s+2}$. This implies that the probability that all of the $s+1$ events - namely those specified by (1) and (2) - occur simultaneously is greater than $1-\rho$. It suffices to show that if $Y \subseteq[0,1]$ is such that $\lambda(Y)<\lambda(U) \delta(\epsilon, k)$ for each $i<k$ then

$$
\lambda\left(\bigcap_{j<k}\left(\bigcup_{n<s}\left(\left(F_{j}\left\lceil P_{n}\right)^{-1} \bigcup_{l<m}\left[\frac{l}{m}, \frac{l+X_{l}}{m}\right]\right) \cup\left(U \backslash C_{j}\right)\right) \cap\left[\Delta_{p}^{i}, \Delta_{p}^{i+1}\right] \backslash Y\right) \geq \frac{\mu}{2 p}\right.
$$

for each $i<p$.

To see this fix $i<p$ and note that

$$
\lambda\left(\bigcap_{j \in k}\left(F_{j}\left\lceil P_{n}\right)^{-1} \bigcup_{i \in m}\left[\frac{i}{m}, \frac{i+X_{i}}{m}\right] \backslash Y\right) \geq \mu \lambda\left(P_{n}\right)-\delta \lambda(U)\right.
$$

and observe that $\left|\left\{n<s: P_{n} \subseteq\left[\Delta_{p}^{i}, \Delta_{p}^{i+1}\right]\right\}\right| \leq 2^{k}$ and so

$$
\begin{gathered}
\lambda\left(\bigcap_{j<k}\left(\bigcup_{n<s}\left(F_{j} \uparrow P_{n}\right)^{-1} \bigcup_{l<m}\left[\frac{l}{m}, \frac{l+X_{l}}{m}\right]\right) \cap\left[\Delta_{p}^{i}, \Delta_{p}^{i+1}\right] \cup\left(U \backslash C_{j}\right) \backslash Y\right) \\
\geq \sum_{\left\{n<s: P_{n} \subseteq\left[\frac{i}{p}, \frac{i+1}{p}\right]\right\}}\left(\mu \lambda\left(P_{n}\right)-\delta \lambda(U)\right) \geq \mu / p-2^{k} \delta \lambda(U)
\end{gathered}
$$

Since $\delta<\frac{\mu}{p 2^{k+1}}$ it follows that $\frac{\mu \lambda(U)}{p}-2^{k} \delta \lambda(U) \geq \frac{\mu \lambda(U)}{2 p}$ as is required.

Notice that the first paragraph of the proof of Lemma 3.3 shows that the choice of $M \in \mathbb{N}$ in Lemma 3.3 is continuous with respect to the measurable functions $\left\{F_{i}: C_{i} \rightarrow[0,1]\right\}_{i<k} \dot{i}$ In particular, suppose that $\epsilon>0, k \in \mathbb{N}$ and $\delta(\epsilon, k)>0$ are given as in the hypothesis of Lemma 3.3. Suppose furthermore that two families of measurable functions $\left\{F_{i}: C_{i} \rightarrow[0,1]\right\}_{i<k}$ and $\left\{F_{i}^{\prime}: C_{i}^{\prime} \rightarrow\right.$ $[0,1]\}_{i<k}$ are also given as in Lemma 3.3 such that $\lambda\left(C_{i} \triangle C_{i}^{\prime}\right)$ are all small and that the supremum norms of the $F_{i}-F_{i}^{\prime}$ are also small. Then a single $M$ can be chosen which will satisfy the conclusion of Lemma 3.3 for both families of functions simultaneously. This remark will be used in the proof of Lemma 3.5. 
Lemma 3.4 Let $U \subseteq[0,1]$ be a measurable subset and let $\left\{F_{i}: C_{i} \rightarrow[0,1]\right\}_{i<k}$ be finitely many measurable functions such that $C_{i} \subseteq U$. Then, for any $\epsilon>0$ there is $\delta>0$ and $A \subseteq[0,1]$ such that $\lambda(A)<\epsilon$ and such that

$$
H_{\infty}^{r}\left(\bigcap_{i<k}\left(F_{i}^{-1} A\right) \cup\left(U \backslash C_{i}\right) \backslash Y\right)>\lambda(U)-\epsilon
$$

for any $Y \subseteq[0,1]$ such that $\lambda(Y)<\delta$.

Proof. For each $i<k$ let $\left\{y_{j}^{i}: j<d_{i}\right\}$ enumerate all points $y \in[0,1]$ such that $\lambda\left(F_{i}^{-1}\{y\}\right)>0$ where $d_{i}$ is an initial segment of $\mathbb{N}$ and the possibility $d_{i}=\mathbb{N}$ is not excluded. Let $C_{i}^{\prime}=C_{i} \backslash F_{i}^{-1}\left\{y_{j}^{i}: j \in d_{i}\right\}$ and let $F_{i}^{\prime}=F_{i}\left\lceil C_{i}^{\prime}\right.$. Since $F_{i}^{\prime}$ has small fibers for each $i<k$ it follows from Lemma 3.3 that it is possible to choose $m$ so large that if $\left\{X_{i}\right\}_{i<m}$ are $\{0,1\}$-valued random variables with mean $\epsilon / 2$, then the probability that, for every $Y$ such that $\lambda(Y)<\delta(\epsilon, k) \lambda(U)$,

$$
H^{r}\left(\bigcap_{i<k}\left(F_{i}^{-1} \bigcup_{j \in m}\left[\frac{j}{m}, \frac{j+X_{j}}{m}\right]\right) \cup\left(U \backslash C_{i}\right) \backslash Y\right)>\lambda(U)-\epsilon / 3
$$

and that, simultaneously,

$$
\left.\lambda\left(\bigcup_{j \in m}\left[\frac{j}{m}, \frac{j+X_{j}}{m}\right]\right)\right)<\epsilon / 2
$$

is greater than 0 . Hence there is $A_{0} \subseteq[0,1]$ such that $\lambda\left(A_{0}\right)<\epsilon / 2$ and such that $H^{r}\left(\bigcap_{i<k}\left(\left(\left(F_{i}^{\prime}\right)^{-1} A_{0}\right) \cup\left(U \backslash C_{i}^{\prime}\right) \backslash Y\right)>\lambda(U)-\epsilon\right.$ for any $Y \subseteq[0,1]$ such that $\lambda(Y)<\delta(\epsilon, k) \lambda(U)$. Now let $K \in \mathbb{N}$ be so large that $\lambda\left(F_{i}^{-1}\left\{y_{j}^{i}: j<\right.\right.$ $K\}) \geq \lambda\left(F_{i}^{-1}\left\{y_{j}^{i}: j \in d_{i}\right\}\right)-\delta(\epsilon, k) \lambda(U) / 2 k$ for each $i<k$. Let $A_{1}$ be a finite union of intervals such that $\lambda\left(A_{1}\right)<\epsilon / 2$ and $\left\{y_{j}^{i}: i<k\right.$ and $\left.j<K\right\} \subseteq A_{1}$. Let $A=A_{0} \cup A_{1}$ and let $\delta=\delta(\epsilon, k) \lambda(U) / 2$. If $\lambda(Y)<\delta$, then

$$
\lambda\left(Y \cup\left(\cup\left(C_{i}^{\prime} \cup F_{i}^{-1}\left\{y_{j}^{i}: j<K\right\}\right)\right)\right)<\delta(\epsilon, k) \lambda(U) .
$$

Hence $H_{\infty}^{r}\left(\bigcap_{i<k}\left(\left(F_{i}^{-1} A\right) \cup\left(U \backslash C_{i}\right) \backslash Y\right)\right.$ is at least as big as $H^{r}\left(\bigcap_{i<k}\left(\left(F_{i}^{-1} A_{0}\right) \cup\right.\right.$ $\left.\left(F_{i}^{-1}\left\{y_{j}^{i}: j \in K\right\}\right) \backslash Y\right)$ which is greater than $\lambda(U)-\epsilon$.

Lemma 3.5 Suppose that $C \subseteq[0,1]^{2}$ is measurable and that $F: C \rightarrow[0,1]$ is a measurable function such that $F_{x}$ has small fibers for each $x \in[0,1]$. Let 
$\mu>0$. Then, for all $\epsilon>0$ there is $A \subseteq[0,1]$ such that $\lambda(A)<2 \mu$ and such that the Lebesgue measure of the set of all $x$ in $[0,1]$ such that

$$
(\forall Y \subseteq[0,1])\left(\lambda(Y)<\delta(\mu, 1) / 2 \Rightarrow H_{\infty}^{r}\left(\left(F_{x}^{-1} A\right) \cup\left([0,1] \backslash C_{x}\right) \backslash Y\right)>1-\mu\right)
$$

is at least $1-\epsilon$.

Proof. Let $\left\{X_{i}\right\}_{i \in \mathbb{N}}$ be a sequence of mutually independent random variable with mean $\mu$. Next, choose a compact subset $W \subseteq C$ such that

- $\lambda(C \backslash W)<\frac{\epsilon \delta(\mu, 1)}{4}$

- $F \uparrow W$ is continuous

- the mapping from $[0,1]$ to $[0,1]$ defined by $x \mapsto \lambda\left(W_{x}\right)$ is continuous

Let $F^{\prime}=F\left\lceil W\right.$. By Lemma 3.3, since each $F \uparrow W_{x}$ has small fibers, and by appealing to Lusin's Theorem,there is $M_{x} \in \mathbb{N}$ such that for all $m>M_{x}$ the probability that

$$
H^{r}\left(\left(F\lceil W)_{x}^{-1} \cup_{j \in m}\left[\frac{j}{m}, \frac{j+X_{j}}{m}\right]\right) \cup\left([0,1] \backslash W_{x}\right) \backslash Y\right)>1-\mu
$$

for any $Y \subseteq[0,1]$ such that $\lambda(Y)<\delta(\mu, 1)$ is greater than $1-\epsilon^{2}$. Since both of the mappings $F$ and $x \mapsto \lambda\left(W_{x}\right)$ are continuous, the proof of Lemma 3.3 shows that the integer $M_{x}$ is valid on a neighborhood of $x$. By compactness, it is possible to find a single $M$ such that for all $m>M$ and for any $x \in[0,1]$ the probability that

$$
H^{r}\left(\left(F\lceil W)_{x}^{-1} \cup_{j \in m}\left[\frac{j}{m}, \frac{j+X_{j}}{m}\right]\right) \cup\left([0,1] \backslash W_{x}\right) \backslash Y\right)>1-\mu
$$

for any $Y \subseteq[0,1]$ is greater than $1-\epsilon^{2}$.

Now let $m>M$ be so great that the probability that $\lambda\left(\cup_{j<m}\left[\frac{j}{m}, \frac{j+X_{j}}{m}\right]\right)<$ $2 \mu$ is greater than $1-2 \epsilon$. Define

$$
\Gamma\left(X_{0}, X_{1}, \ldots, X_{m}\right)
$$

to be the Lebesgue measure of the set of all $x \in[0,1]$ such that

$$
\left.H^{r}\left((F \uparrow W)_{x}^{-1} \cup_{j \in m}\left[\frac{j}{m}, \frac{j+X_{j}}{m}\right]\right) \cup\left([0,1] \backslash W_{x}\right) \backslash Y\right)>1-\mu
$$

for any $Y \subseteq[0,1]$ such that $\lambda(Y)<\delta(\mu, 1)$. The first step is to estimate

$$
\alpha_{m}=\sum_{X_{0}=0}^{1} \sum_{X_{1}=0}^{1} \ldots \sum_{X_{m}=0}^{1} \Gamma\left(X_{0}, X_{1}, \ldots, X_{m}\right) \prod_{i=0}^{m} \mu^{X_{i}}(1-\mu)^{1-X_{i}},
$$


the average value of $\Gamma\left(X_{0}, X_{1}, \ldots, X_{m}\right)$. To this end, let

$$
\Lambda_{x}\left(X_{0}, X_{1}, \ldots, X_{m}\right) \in\{0,1\}
$$

be defined to be 1 if and only if

$$
\left.H^{r}\left((F \uparrow W)_{x}^{-1} \bigcup_{j \in m}\left[\frac{j}{m}, \frac{j+X_{j}}{m}\right]\right) \cup\left([0,1] \backslash W_{x}\right) \backslash Y\right)>1-\mu
$$

for any $Y \subseteq[0,1]$ such that $\lambda(Y)<\delta(\mu, 1)$. Observe that $\alpha_{m}$ is equal to

$$
\begin{gathered}
\sum_{X_{0}=0}^{1} \sum_{X_{1}=0}^{1} \ldots \sum_{X_{m}=0}^{1}\left(\int_{x \in[0,1]} \Lambda_{x}\left(X_{0}, X_{1}, \ldots, X_{m}\right) d x\right) \prod_{i=0}^{m} \mu^{X_{i}}(1-\mu)^{1-X_{i}}= \\
\int_{x \in[0,1]}\left(\sum_{X_{0}=0}^{1} \sum_{X_{1}=0}^{1} \ldots \sum_{X_{m}=0}^{1} \Lambda_{x}\left(X_{0}, X_{1}, \ldots, X_{m}\right) \prod_{i=0}^{m} \mu^{X_{i}}(1-\mu)^{1-X_{i}}\right) d x
\end{gathered}
$$

However, notice that

$$
\sum_{X_{0}=0}^{1} \sum_{X_{1}=0}^{1} \ldots \sum_{X_{m}=0}^{1} \Lambda_{x}\left(X_{0}, X_{1}, \ldots, X_{m}\right) \prod_{i=0}^{m} \mu^{X_{i}}(1-\mu)^{1-X_{i}}
$$

is just the probability that

$$
H^{r}\left(\left(F\lceil W)_{x}^{-1} \cup_{j \in m}\left[\frac{j}{m}, \frac{j+X_{j}}{m}\right]\right) \cup\left([0,1] \backslash W_{x}\right) \backslash Y\right)>1-\mu
$$

for any $Y \subseteq[0,1]$ such that $\lambda(Y)<\delta(\mu, 1)$ and the choice of $m$ guarantees that this probability is greater than $1-\epsilon^{2}$. Hence $\alpha_{m} \geq 1-\epsilon^{2}$.

Now let $p$ be the probability that $\Gamma\left(X_{0}, X_{1}, \ldots, X_{m}\right) \geq 1-\epsilon / 2$. Obviously, $p+(1-p)(1-\epsilon / 2) \geq \alpha_{m} \geq 1-\epsilon^{2}$. Solving for $p$ yields that $p \geq 1-2 \epsilon$. Since $m$ was chosen so large that the probability that $\lambda\left(\cup_{j<m}\left[\frac{j}{m}, \frac{j+\bar{X}_{j}}{m}\right]\right)<2 \mu$ is greater than $1-2 \epsilon$, there is at least one $A$ which is formed by taking the union of no more than $2 \mu m$ intervals $\left[\frac{i}{m}, \frac{i+1}{m}\right]$ - and hence $\lambda(A)<2 \mu-$ such that $\lambda(Z)>1-\epsilon / 2$ where $Z$ is the set of all $x \in[0,1]$ such that

$$
H^{r}\left(\left(\left(F\lceil W)_{x}^{-1} A\right) \cup\left([0,1] \backslash W_{x}\right) \backslash Y\right)>1-\mu\right.
$$

for any $Y \subseteq[0,1]$ such that $\lambda(Y)<\delta(\mu, 1)$.

Now notice that $\lambda\left(\left\{x \in[0,1]: \lambda\left(C_{x} \backslash W_{x}\right) \geq \frac{\delta(\mu, 1)}{2}\right\}\right)<\epsilon / 2$ and so it suffices to observe that if $x \in Z \backslash\left\{x \in[0,1]: \lambda\left(C_{x} \backslash W_{x}\right) \geq \frac{\delta(\mu, 1)}{2}\right\}$ and $\lambda(Y)<\frac{\delta(\mu, 1)}{2}$ then $\lambda\left(Y \cup\left(C_{x} \backslash W_{x}\right)\right)<\delta(\mu, 1)$ and so $H^{r}\left((F)_{x}^{-1} A \cup\left([0,1] \backslash C_{x}\right) \backslash Y\right) \geq H^{r}\left((F)_{x}^{-1} A \cup\left([0,1] \backslash W_{x}\right) \backslash\left(C_{x} \cup Y\right)\right)>1-\mu$. 
Theorem 3.1 Let $F:[0,1]^{2} \rightarrow[0,1]$ be a continuous function and suppose that $\epsilon>0$. Then there is $A \subseteq[0,1]$ such that $\lambda(A)<\epsilon$ and

$$
H_{\infty}^{r}\left\{x \in[0,1]: H_{\infty}^{r}\left(F_{x}^{-1} A\right)>1-\epsilon\right\}>1-\epsilon .
$$

Proof. Let $B=\left\{(x, y) \in[0,1]^{2}: \lambda\left(F_{x}^{-1}\{y\}\right)>0\right\}$ and note that

$$
B=\left\{(x, y) \in[0,1]^{2}:(\exists K \text { compact })\left(\lambda(K)>0 \text { and } K \subseteq F_{x}^{-1}\{y\}\right\}\right.
$$

and, because $F$ is continuous, the relation $K \subseteq F_{x}^{-1}\{y\}$ is Borel. Moreover, so is the statement $\lambda(K)>0$ and so the set $B$ is $\Sigma_{1}^{1}$ and hence, measurable. Let $B^{*}=F^{-1} B$. Since $B^{*}$ is clearly measurable, it follows that so is $C=$ $[0,1]^{2} \backslash B^{*}$. Now let $\left\{f_{j}: j \in d\right\}$ enumerate a maximal collection of functions such that

- $f_{j}: C_{j} \rightarrow[0,1]$ where $C_{j} \subseteq[0,1]$ is compact

- $f_{j}$ is continuous

- $f_{j} \subseteq B\left(f_{j}\right.$ is, of course, identical to the graph of $\left.f_{j}\right)$

- if $x \in C_{j} \cap C_{n}$, then $f_{j}(x) \neq f_{n}(x)$

- $\int_{C_{j}} \lambda\left(F_{x}^{-1}\left\{f_{j}(x)\right\}\right) d x>0$.

The first thing to notice is that such a family must be countable. To see this let $E_{j}=\left\{(x, y) \in[0,1]^{2}: F(x, y)=f_{j}(x)\right\}$. If $n \neq j$, then $E_{j} \cap E_{n}=\emptyset$ and, moreover,

$$
\lambda\left(E_{j}\right)=\int_{C_{j}} \lambda\left(F_{x}^{-1}\left\{f_{j}(x)\right\}\right) d x>0
$$

for any $j<d$. Hence the family of sets $E_{j}$ is countable and so it can be assumed that $d$ is am initial segment of $\mathbb{N}$.

Next, it must be shown that

$$
\left.\sum_{j<d} \int_{C_{j}} \lambda\left(F_{x}\right)^{-1}\left\{f_{j}(x)\right\}\right) d x=\lambda\left(B^{*}\right)
$$

so suppose not. Then it must be that $\lambda\left(B^{*} \backslash \cup_{j<d} E_{j}\right)>0$. Since each $f_{j}$ is continuous and $d \leq \mathbb{N}$, it follows that $B \backslash\left(\cup_{j \in d} f_{j}\right)$ is $\Sigma_{1}^{1}$. Hence it is possible to use the von Neumann selection theorem (Exercise 4E.9 in [3] or Theorem 18.1 in [2]) to find a function $f$ such that the domain of $f$ is

$$
D=\left\{x \in[0,1]:(\exists y \in[0,1])\left((x, y) \in B \backslash\left(\cup_{j \in d} f_{j}\right)\right)\right\}
$$


and $f$ is measurable. Since $D$ is also equal to

$$
\left\{x \in[0,1]:(\exists y \in[0,1])(x, y) \in B^{*} \backslash\left(\cup_{j \in d} E_{j}\right\}\right)
$$

it must be that $\lambda(D)>0$. Hence

$$
\int_{D} \lambda\left(F_{x}^{-1}\{f(x)\}\right) d x>0
$$

because $\lambda\left(F_{x}^{-1}\{f(x)\}\right)>0$ for each $x \in D$. Finally, by using Lusin's Theorem, it is possible to find a compact set, $D^{\prime}$ such that $f \uparrow D^{\prime}$ is continuous and $\int_{D^{\prime}} \lambda\left(F_{x}^{-1}\{f(x)\}\right) d x>0$. This contradicts the maximality of the family $\left\{f_{j}\right.$ : $j<d\}$.

It is therefore possible to find $K \in \mathbb{N}$ such that

$$
\sum_{j<K} \int_{C_{j}} \lambda\left(F_{x}^{-1}\left\{f_{j}(x)\right\}\right) d x>\lambda\left(B^{*}\right)-\frac{\epsilon \delta(\epsilon / 4,1)}{4} .
$$

In particular, if $S$ is defined to be

$$
\left\{x \in[0,1]: \lambda\left(\left(B^{*}\right)_{x} \backslash F_{x}^{-1}\left\{f_{j}(x): j<K\right\}\right) \geq \delta(\epsilon / 4,1) / 2\right\},
$$

then $\lambda(S)<\epsilon / 2$ and hence, if $U$ is defined to be $[0,1] \backslash S$, then $\lambda(U)>1-\epsilon / 2$. Using Lemma 3.4 applied to $\left\{f_{j}: j<K\right\}, U$ and $\epsilon / 2$, there is $A_{0} \subseteq[0,1]$ such that $\lambda\left(A_{0}\right)<\epsilon / 2$ and there is some $\delta>0$ such that if

$$
W=U \cap\left(\bigcap_{i<K}\left(f_{j}^{-1} A_{0}\right) \cup\left(U \backslash C_{j}\right)\right)
$$

then $H_{\infty}^{r}(W \backslash Y)>\lambda(U)-\epsilon / 2$ for any $Y \subseteq[0,1]$ such that $\lambda(Y)<\delta$.

Now note that $\left(F\lceil C)_{x}\right.$ has small fibers for all $x$. Use Lemma 3.5 to choose $A_{1} \subseteq[0,1]$ such that $\lambda\left(A_{1}\right)<\epsilon / 2$ and such that the Lebesgue measure of the set $Z$ consisting of all $x$ in $[0,1]$ such that

$$
(\forall Y \subseteq[0,1])\left(\lambda(Y)<\delta(\epsilon / 4) / 2 \Rightarrow H_{\infty}^{r}\left(\left(F_{x}^{-1} A_{1}\right) \cup\left([0,1] \backslash C_{x}\right) \backslash Y\right)>1-\epsilon\right)
$$

is at least $1-\delta$. Now let $A=A_{0} \cup A_{1}$.

Notice that $H_{\infty}^{r}(W \cap Z)>\lambda(U)-\epsilon / 2>1-\epsilon$. Hence, it suffices to show that if $x \in W \cap Z$ then $H_{\infty}^{r}\left(F_{x}^{-1} A\right)>1-\epsilon$. To see this recall that if $x \in W \subseteq U$, then the Lebesgue measure of $Y(x)=\left(B^{*}\right)_{x} \backslash F_{x}^{-1}\left\{f_{j}(x): j<K\right\}$ is less than $\delta(\epsilon / 4,1) / 2$. Because $x \in Z$, it follows that $H_{\infty}^{r}\left(\left(F_{x}^{-1} A_{1}\right) \cup\left([0,1] \backslash C_{x}\right) \backslash Y(x)\right)>$ $1-\epsilon$. But,

$\left([0,1] \backslash C_{x}\right) \backslash Y(x)=B_{x}^{*} \backslash\left(\left(B^{*}\right)_{x} \backslash F_{x}^{-1}\left\{f_{j}(x): j<K\right\}\right)=F_{x}^{-1}\left\{f_{j}(x): j<K\right\}$ 
and hence $\left(F_{x}^{-1} A_{1}\right) \cup\left([0,1] \backslash C_{x}\right) \backslash Y(x)=\left(F_{x}^{-1} A_{1}\right) \cup F_{x}^{-1}\left\{f_{j}(x): j<K\right\}$. However, if $y \in F_{x}^{-1}\left\{f_{j}(x): j<K\right\}$, then $F_{x}(y)=f_{j}(x)$ for some $j<K$. It follows that $x \notin U \backslash C_{j}$ and hence $x \in f_{j}^{-1} A_{0}$ because $x \in W$. Therefore $F_{x}(y)=f_{j}(x) \in A_{0}$ and, since $y$ was arbitrary, it may be concluded that $F_{x}^{-1}\left\{f_{j}(x): j<K\right\} \subseteq F_{x}^{-1} A_{0}$. Since $F_{x}^{-1} A \supseteq F_{x}^{-1} A_{1} \cup F_{x}^{-1} A_{0}$ this finishes the proof.

It is worth noting that Theorem 3.1 immediately implies that if $F:[0,1] \rightarrow$ $[0,1]$ is a continuous function and $\epsilon>0$ then there is $A \subseteq[0,1]$ such that $\lambda(A)<\epsilon$ and $H_{\infty}^{r}\left(F^{-1} A\right)>1-\epsilon-$ simply consider the function $F^{*}$ : $[0,1]^{2} \rightarrow[0,1]$ defined by $F^{*}(x, y)=F(y)$.

Finally, it should be remarked that Theorem 3.1 has obvious generalizations to higher dimensions which can be proved using induction on the dimension by using the techniques of this paper. However, the strengthened requirements of the induction clutter the main argument considerably. In particular, it is necessary to prove analogous versions of Theorem 3.1 for finitely many functions as well as with a parameter corresponding to the set $U$ of Lemma 3.4.

\section{References}

1. Lennart Carleson, Selected problems on exceptional sets, Mathematical Studies,vol. 13, Van Nostrand, New York, 1967.

2. Alexander S. Kechris, Classical descriptive set theory, Graduate Texts in Mathematics, vol. 156, Springer, New York, 1995.

3. Y. N. Moschovakis, Descriptive set theory, Studies in Logic, vol. 100, NorthHolland, Amsterdam, 1980.

4. J. Steprāns, Cardinal invariants associated with Hausdorff capacities, Proceeding of the BEST Conferences 1-3, Contemporary Mathematics, 192, AMS, 157-184. 